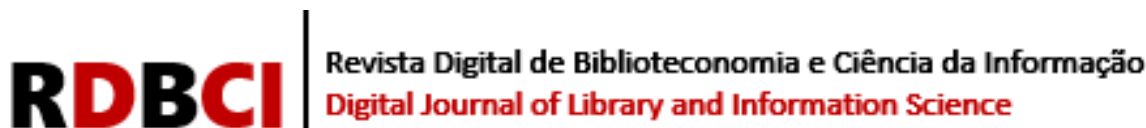

\section{$\mathbf{I S} \mathbf{N}_{1678}$ \\ $678-765 x$ \\ do \\ 10.20396/rdbci.v19ioo.8664141 \\ Contexto das publicações científicas em periódicos indexados regionais versus publicações globais}

(c) (i) $\mathrm{BY}$

ARTIGO

\author{
Israel Barrutia Barreto ${ }^{1}$ iD https://orcid.org/0000-0001-5115-3371 \\ Haiber Policarpio Echevarria Rodríguez ${ }^{2}$ iD https://orcid.org/0000-0002-5216-175X \\ Wilmer Ortega Chávez ${ }^{3}$ iD https://orcid.org/0000-0003-2738-8060 \\ ${ }^{1}$ Innova Scientific, Lima, Peru / e-mail: israel2obarrutia@gmail.com \\ ${ }^{2}$ Universidade Nacional Hermilio Valdizan Huanuco, Lima, Peru / e-mail: haiberechevarriarod@gmail.com \\ ${ }^{3}$ Universidade Intercultural Nacional da Amazônia, Lima, Peru / e-mail: wilmerortegachavezı234@gmail.com
}

RESUMO

Introdução/Objetivo: O objetivo deste article foi aprofundar os resultados obtidos na revisão bibliográfica de referências, em publicações de alto impacto, colocadas em periódicos indexados em bases de dados de alto nível, tais como: Scopus e Web of Science (WoS) comparados a publicações das mesmas. impacto, mas no contexto global. Método/Resultados: Utilizou-se o método de pesquisa (descritivo-documental), tomando como referência artigos relacionados ao tema. Portanto, os resultados mostraram que no Peru e no Equador, países que estão subindo nessas bases de dados, temos apenas 14 periódicos peruanos indexados no WoS e 9 no Scopus, para o Equador seus periódicos indexados no WoS chegam a 20, enquanto para Scopus, é reduzido a apenas 2 revistas. Conclusão: De acordo com esses dados, foram discutidas as medidas tomadas pelos dois países para aumentar sua proliferação científica e em que medida o Estado contribui para esse aprimoramento acadêmico-profissional.

PALAVRAS-CHAVE

Análise bibliométrica. América Latina. Peru. Produção científica. Revistas indexadas.

\section{Context of scientific publications in regionally indexed journals versus global publications}

\begin{abstract}
Introduction/Objective: The objective of this article was to deepen the results obtained in the bibliographic review of references, in high impact publications, placed in journals indexed in high level databases, such as: Scopus and Web of Science (WoS) compared to publications of the same. impact, but in the global context. Method/Results: The research method (descriptive-documental) was used, taking as a reference articles related to the theme. Therefore, the results showed that in Peru and Ecuador, countries that are going up in these databases, we have only 14 Peruvian journals indexed in WoS and 9 in Scopus, for Ecuador their journals indexed in WoS reach 20, while for Scopus, is reduced to just 2 magazines. Conclusion: According to these data, the measures taken by the two countries to increase their scientific proliferation and the extent to which the State contributes to this academic-professional.
\end{abstract}

KEYWORDS

Bibliometric analysis. Latin America. Perú. Scientific production. Indexed journals.

RDBCl: Rev. Dig. Bibliotec e Ci. Info. / RDBCl: Dig. J. of Lib. and Info. Sci. | Campinas, SP | v.19| e021009 | 2021 


\section{1 Revista Digital de Biblioteconomia e Ciência da Informação Digital Journal of Library and Information Science}

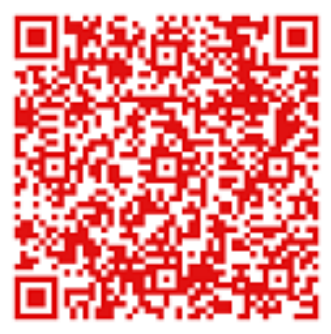

JITA: BB. Bibliometric methods. 


\section{$\mathrm{RDBCI} \mid=0$}

\section{INTRODUÇÃO}

O impacto do desenvolvimento técnico-científico nas ciências sociais tem promovido a regulação da produção científica baseada em critérios de qualidade, determinando a visibilidade do conhecimento e a sua disponibilidade para a formação, investigação científica e sua aplicação ao ambiente social (MARDONES, 2016). Na última década, tanto o Peru quanto o Equador realizaram constantes esforços para aumentar sua produção científica e, ao mesmo tempo se posicionar em bases de dados de alto impacto, tais como, WoS (WEB OF SCIENCE) e SCOPUS, este esforço teve origem na preocupação dos profissionais e sua busca por posicionamento e reconhecimento a nível global.

Nas últimas duas décadas, aumentou o interesse com respeito a como algumas universidades de pesquisa tiveram um impacto direto nas atividades econômicas regionais e no crescimento, embora, o papel da pesquisa básica para a maioria das universidades tenha permanecido forte, intensificou-se a pressão para expandir suas missões de modo a incluir o apoio aos esforços de desenvolvimento econômico local e regional (TEJEDOR-ESTUPINAN e TEJEDOR-ESTUPINAN, 2019). Consequentemente, muitas universidades desenvolveram a sua missão de pesquisa científica básica, desde a produção de conhecimentos científicos até ao intercâmbio e disseminação do conhecimento com as indústrias locais, mediante a participação ativa no desenvolvimento econômico local (CASTRO-RODRÍGUEZ, 2019; GUERRA et al., 2013; PERDOMO et al. 2020; SANCHO et al., 2006; TAYPE-RONDÁN et al., 2014).

Nesse sentido, a pesquisa de CRESPO-GASCÓN et al. (2019), demonstrou que a maioria das universidades, contribuiu para o desenvolvimento econômico local e regional, através das diversas funções oferecidas, sendo estas as seguintes: criação de conhecimento, criação de capital humano, transferência do conhecimento existente, inovação tecnológica, investimento de capital, liderança regional, produção de infraestruturas de conhecimento e influência no ambiente regional. As atividades universitárias, principalmente as centradas na área do conhecimento, tiveram efeitos positivos substanciais numa variedade de medidas de progresso econômico regional (ANGULO et al., 2008; DRUCKER e GOLDSTEIN, 2007; GONZÁLEZ-ARGOTE et al., 2016).

De acordo com as abordagens anteriores, foi possível estabelecer que, a produção científica e o impacto que estas publicações geram tanto a nível regional como global, estão fortemente relacionados com o desenvolvimento econômico do seu país de origem, uma vez que estes novos conhecimentos ou propostas de novos estudos estão centrados no desenvolvimento industrial de cada país, conseguindo desta forma um arranque dos mesmos e resultando em melhorias para a economia nacional. Portanto, é transcendental que estas investigações e posteriores publicações de elevado impacto global também sejam corrigidas pelas entidades correspondentes do Governo Nacional, através de políticas públicas que conduzam a uma melhoria na qualidade atual das publicações e, por conseguinte, das próprias revistas. O contraste destas possíveis novas políticas públicas, poderia resultar em baixa produtividade, principalmente devido à burocratização, o que causa prolongamento e perda de tempo no processo de pagamento de subsídios pelo Estado (COCCIA and ROLFO, 2007; RIOS-GONZÁLEZ, 2016).

Embora a América Latina seja um continente que conta com um extenso território, sua produção científica ainda é de baixo impacto. Isto, até o momento, impede-o de estar numa boa posição, dentro do ranking da produção científica global. Segundo RAIHER (2010) os fatores mais relevantes são: baixo investimento do Estado, o incipiente investimento privado em favor de atividades científico-tecnológicas, o pequeno número de profissionais envolvidos inteiramente na área de pesquisa e desenvolvimento tecnológico, e o elevado custo dos 


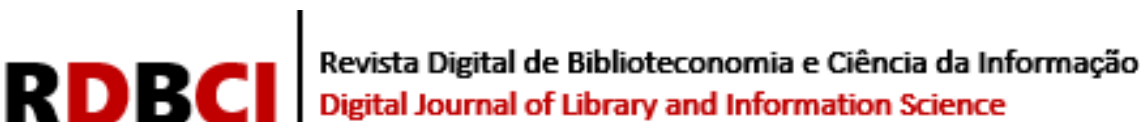

equipamentos e materiais científicos. Mas também deve ser mencionado que, atualmente na América Latina, os temas com maior produtividade estão localizados na área de saúde pública, enfermidades infecciosas, cirurgia, neurologia, cardiologia e medicina cardiovascular, ou seja, os campos com maior visibilidade estão principalmente relacionados com problemas de saúde global, doenças crónicas e emergentes. Os países mais prolíficos são o Brasil, México e Argentina, embora aqueles com maior impacto e maior colaboração sejam os seguintes: Peru, Porto Rico e Argentina (ZACCA-GONZÁLEZ et al., 2015; CRUZ-RAMÍREZ et al., 2014). De acordo com estes dados, podemos então notar que embora o Peru não seja atualmente um dos países com maior proliferação científica, está registado como um dos países de maior impacto, especialmente na área da medicina. Por conseguinte, o principal objetivo do Peru deve ser o de se concentrar na obtenção de uma maior produtividade dos artigos que mantêm a sua elevada qualidade. Enquanto no Equador o panorama é totalmente diferente, mostrando uma elevada produtividade na última década, mas ainda não gerando um impacto totalmente elevado.

Para o Equador, a sua produção científica em relação ao resto dos países latinoamericanos tem sido historicamente mínima, mostrando uma visível falta de cultura científica e uma gestão política inadequada para a promoção da investigação científica, bem como uma notória negligência na priorização do desenvolvimento científico, nos centros universitários (MOREIRA-MIELES et al., 2020). Contudo, durante a última década, o governo implementou diversas políticas para ajudar a remediar este panorama atual. Os resultados mostram que o Equador aumentou a sua produção científica em 5,16 vezes nos últimos anos, o que excede o crescimento latino-americano. As colaborações internacionais excedem $80 \%$ das publicações equatorianas, principalmente com os seguintes países: Estados Unidos, Espanha, Inglaterra, Alemanha, França, Brasil e Colômbia (CASTILLO e POWEL, 2019).

Por outro lado, na produção científica peruana, uma das principais desvantagens do posicionamento do país no ranking das revistas de alto impacto global deve-se a que os investigadores peruanos com fatores de alto impacto provêm principalmente de universidades estrangeiras e publicam artigos académicos como coautores com outros acadêmicos peruanos nativos. Em relação às instituições académicas peruanas, apenas algumas têm um impacto elevado na investigação (6 em WOS e 6 em SCOPUS), enquanto um grupo maior atingiu um impacto médio na investigação (10 em WOS e 14 em SCOPUS) e a grande maioria das instituições mostrou um baixo impacto na investigação (Huamaní et al., 2013).

No Peru, o órgão regulador do setor nacional de investigação é o Conselho Nacional para a Ciência, Tecnologia e Inovação Tecnológica (CONCYTEC), que reconhece e regista as revistas científicas peruanas, para a sua posterior indexação no sistema (CASTRORODRÍGUEZ et al., 2019). Embora o Peru seja um país que está atualmente desenvolvendo sua linha de educação e pesquisa, ainda não atinge os resultados esperados, isto pode ter como causa a falta de recursos financeiros para a implementação de projetos importantes e a escassez de capital humano qualificado, o que explicaria em grande parte esta realidade Por outro lado, VALLE e SALVADOR (2012), propõem que a produção científica e o avanço dos projetos de pesquisa e desenvolvimento tecnológico sejam transformados em conhecimento proveitoso, útil para elaborar novos produtos e diversificar a economia. A diversificação da economia aumenta a complexidade econômica do país e permite sustentar o crescimento econômico a médio prazo através do aumento da produtividade. Daí a importância de promover o investimento no campo da Ciência, Tecnologia e Inovação Tecnológica, num cenário pouco encorajador em que estamos na sua maioria atrasados em relação a outros países da região com características semelhantes. Considerando os resultados disponíveis até 2017 e considerando apenas três das principais bases de dados a nível mundial e regional, a saber, Scopus, Scielo e Índice de Citação de Fontes Emergentes - ESCI / WoS, o Peru tem apenas 29 revistas universitárias indexadas, de mais de 140 universidades nacionais, das quais apenas 14 são acessíveis ao público, 3 são 


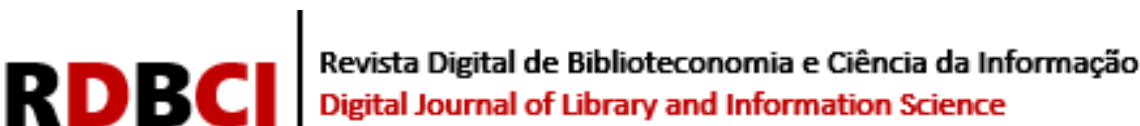

indexadas no Scopus, 24 no Scielo e 14 no ESCI. O Peru continua com menor produtividade em relação ao Equador, embora sua qualidade seja maior, porém, tem sido insuficiente em termos de buscar um bom posicionamento de suas revistas e gerar um alto impacto com as publicações nelas feitas (CASIMIRO URCOS, 2020; GARCIA-BEREGUIAIN, 2019; ZUÑIGA,2000).

O problema que ambos os países enfrentam é o baixo impacto gerado pelas suas publicações em comparação com outros países, que também com este alto impacto, conseguem um melhor posicionamento das suas revistas em bases de dados com elevados padrões de qualidade, tais como SCOPUS e WOS. Representando no Peru, a sua baixa produtividade científica, como fator fundamental do impacto das suas publicações, embora tenha uma elevada qualidade, de acordo com dados da Scopus (VERA-MONGE et al, 2017; SCIMAGO, 2011). Enquanto que, no Equador, o baixo impacto nas publicações científicas deve-se ao fato de que antes das duas últimas décadas, as suas políticas públicas eram mínimas, causando uma diminuição na qualidade das suas publicações. Apesar de atualmente o seu crescimento, em relação às suas revistas indexadas em SCOPUS e WOS, em comparação com outros países da América Latina, ter sido abismalmente superior, ainda não atinge um bom posicionamento nas mesmas plataformas, já mencionadas.

Portanto, o objetivo desta investigação foi aprofundar os resultados obtidos na revisão sistemática de referências, sobre publicações de alto impacto, colocadas em revistas indexadas na base de dados de alto nível, tais como: SCOPUS e Web of Science (WOS) comparados a publicações de mesmo impacto, mas no contexto global.

Assim mesmo, se busca fazer referência ao impacto das publicações de ambos os países em relação a outros países da América Latina e, ao mesmo tempo, situá-los no contexto global. No caso do Equador, procurou-se encontrar os progressos feitos por este país na última década, tanto em nível de produtividade como de qualidade, relacionando-o diretamente com as políticas públicas tomadas pelo Governo equatoriano. No caso do Peru, a linha de pesquisa foi a mesma, acrescentando seu âmbito em relação a outros países, as suas atuais políticas públicas em relação ao sector da investigação e também possíveis recomendações baseadas na coleta de tais dados

Para o Peru, os registos do último censo do setor de pesquisa em 2016, determinado pelo CONCYTEC (Conselho Nacional de Ciência, Tecnologia e Inovação Tecnológica) foram utilizados como fonte de dados, e para o país irmão Equador, as suas referências foram retiradas da SENESCYT (Secretaria do Ensino Superior, Ciência, Tecnologia e Inovação). Estes dados foram contrastados e referenciados em relação ao panorama mundial, a partir da presença de artigos em revistas indexadas de alto impacto.

\section{METODOLOGIA}

A presente pesquisa foi do tipo documental e descritiva, uma vez que os dados selecionados foram consultados a partir de várias fontes documentais, pelo que a informação localizada no período 2012-2018 corresponde à base de dados CONCYTEC, para coletar dados estatísticos peruanos; SENESCYT, para resultados do Equador e a própria base de dados SCOPUS, para comparar a produção científica total na América Latina com respeito aos dois países anteriormente especificados. A amostragem foi não probabilística, pois a base de dados selecionada se baseava no seu livre acesso e capacidade de gestão, bem como na sua veracidade. Para a coleta de dados, foi utilizado o programa Excel para delimitar os dados e criar uma tabela comparativa em relação aos países com os quais esta investigação foi realizada. Na sequência, 


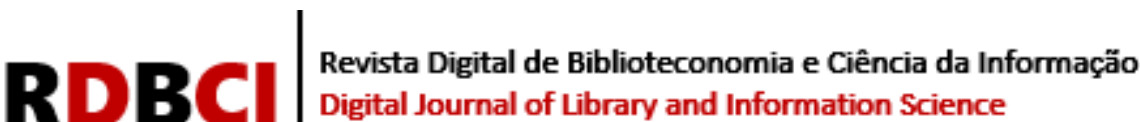

detalharemos as fontes utilizadas e alguns dados relevantes para a apresentação subsequente dos resultados estatísticos.

\section{FONTES}

\subsection{CONCYTEC - Perú}

Durante o ano de 2014, as despesas atribuídas pelos centros de pesquisas e desenvolvimento tecnológico igualaram o número de 438 milhões de soles, o que constitui 0,08\% do PIB nacional para o mesmo ano. Em 2015, o número aumentou para 518 milhões de soles. No entanto, como proporção do PIB, manteve-se em 0,08\%. No panorama mundial, esta cifra foi mínima, em correspondência com as outras seções da Aliança do Pacífico e América Latina, o que indica o fraco investimento que o setor da investigação tem no território peruano. Durante o ano de 2015, o total de peritos dedicados inteiramente à geração de Pesquisa e Desenvolvimento (P \& D) foi igual a 5.408 pessoas, mostrando um aumento de $13,1 \%$, em relação ao ano anterior, que somou um total de 4.708 profissionais. De acordo com a categoria de pessoal, 62,4\% do total de profissionais dedicados à $\mathrm{P} \& \mathrm{D}$ foram registados como investigadores; $22,1 \%$ estavam localizados na categoria de técnicos e os restantes $15,5 \%$ foram reconhecidos como pessoal de apoio.

A nível mundial, o Peru está em desvantagem em comparação com nações equivalentes na região, devido ao número de pesquisadores. Assim, no Peru há apenas 0,2 pesquisadores por cada mil participantes da sua População Economicamente Ativa (PEA), que está abaixo da média dos restantes países da América Latina $(1,3 \%)$ e 12,7\%, o que está bastante longe da média dos países da OCDE. Em 2015, 31,8\% dos investigadores tinham título de doutor (1.069 com doutorado), o que era muito inferior ao de outros países da América Latina. Por exemplo, em países como Chile e Uruguai esta percentagem era igual a 39,2\% e 64,2\%, respectivamente. Por outro lado, para a Organização para a Cooperação e Desenvolvimento Econômico (OCDE), esta percentagem era de 42,8\%. Do mesmo modo, 27\% dos investigadores pertenciam à área da Engenharia e Tecnologia; $25 \%$ ao sector das Ciências Naturais e $20 \%$ às Ciências Sociais.

De acordo com Estrada-Cuzcano e Alfaro (2019), se consideraram três das principais bases de dados internacionais e regionais, sendo estas SCOPUS, SCIELO e INDEX de Fontes Emergentes - ESCI/WoS, Peru contava com 29 revistas universitárias indexadas, as mesmas que foram publicadas, apenas por 14 universidades (de uma média de mais de 140 universidades de acordo com dados da SUNEDU). De notar que, destas 14 universidades, 11 estão atualmente licenciadas e as restantes estão a meio do processo. Por outro lado, de acordo com a variável de especialidade, foi especificado que Ciências Sociais e Humanas reuniam a maior quantidade de revistas indexadas (13); logo encontrámos o campo das Ciências Biomédicas (8); Ciências Básicas (7); Ciências Empresariais (1) e finalmente no campo da engenharia nenhuma está localizada nestas bases de dados, o que contrasta paradoxalmente com a relevância das Ciências Sociais e Humanas, em comparação com o seu menor apoio e a baixa solvência proporcionada pelos seus projetos. Do mesmo modo, se detalhou que a maioria das revistas indexadas provém de universidades de capital privado (21), frente a 8 universidades gestão pública e que, em relação à sua região de procedência, 10 são de Lima e apenas 4 das províncias.

Finalmente, notou-se que 55\% das revistas globais indexadas em bases de dados como a WOS e SCOPUS, estavam concentradas principalmente em 3 universidades: Pontificia Universidade Católica do Perú (9), seguida da Universidade Nacional Mayor de San Marcos 


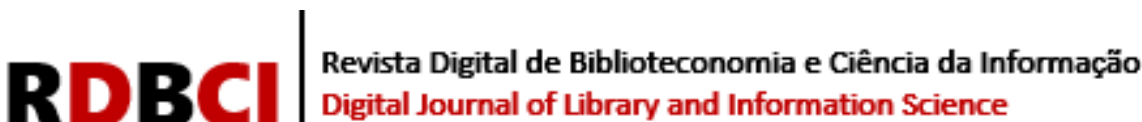

(4) e finalmente Universidade Peruana Cayetano Heredia, com 3 revistas indexadas. A partir desta informação, destacou-se que o campo das Humanidades, é atualmente a especialidade com menos divulgações, mas em total contraste com estes dados, é a que apresenta o maior número de revistas indexadas, igual em marca à Engenharia e Tecnologia ou ultrapassando largamente o campo das Ciências Agrárias; também, ligando ambas investigações acima referidas, deve notar-se que o peso do campo das Ciências Biomédicas, tem até agora, um total de 8 revistas indexadas, superior ao seu número de publicações e, portanto, ainda apresentou baixo impacto internacional. De outro ângulo, os centros universitários com o maior número de artigos indexados na base de dados SCOPUS, entre 2012-2017 são: a Universidade Peruana Cayetano Heredia (UPCH) com 1930 publicações; depois a Universidade Nacional Mayor de San Marcos (UNMSM) com 1430 artigos divulgados; e finalmente a Pontifícia Universidade Católica do Peru (PUCP) que registrou um total de 1356 publicações; o que indicou que apenas nestas três universidades, $55 \%$ das revistas indexadas estavam concentradas.

\subsection{SENESCYT - ECUador}

De acordo com relatórios desse órgão estatal, até ao final de janeiro deste ano, na sequência do plano anual, o governo equatoriano contribuiu para o sector da investigação, especificamente para o SENESCYT, um total de 2,07\% correspondente ao seu PIB nacional, ou seja, a soma total de 4.928.422,51 sucres derivados da produção científica do país. Em relação à América Latina, o país irmão do Equador, durante a última década, tem sido um dos países que mais tem impulsionado seu sector de pesquisa, proporcionando políticas públicas que beneficiam a produtividade e a melhoria da qualidade das publicações indexadas a revistas de alto impacto global. E desta forma conseguiu posicionar-se como o país latino-americano com mais avanços na investigação e desenvolvimento, em relação aos outros países latinoamericanos.

De acordo com o site oficial da SCOPUS, até abril de 2018, o Equador produziu três revistas: "Correo poblacional y de la salud / Centro de Estudios de Población y Paternidad Responsable", que esteve inativo desde 1999, na área de Oncologia e a revista "Ecuatoriana de Neurología", que foi desativada desde 1992 e registrada novamente durante 2015, atualmente. A única revista equatoriana de Neurologia, "Sociedad Ecuatoriana de Neurología", registrada na base de dados SCOPUS, referia-se a 26 revistas publicadas, com publicações trimestrais, até 2017.

\subsection{Base de dados SCOPUS - Panorama da América Latina}

Usando a base de dados SCIMAGO/SCOPUS como referência, conseguimos classificar um grupo de países da América Latina, de acordo com o seu respectivo número de revistas indexadas nessa base de dados, classificando os primeiros 10 países desta lista: Brasil (378); México (114); Chile (102); Colômbia (99); Argentina (64); Venezuela (39); Cuba (25); Peru (9); Equador (2) e Uruguai (1). A existência de revistas indexadas em bases de dados internacionais como SCOPUS num determinado país não só permitiu a publicação de artigos na língua materna dos autores, como também permitiu a publicação de temas de interesse regional e não necessariamente no contexto internacional. Isto tornou-se definitivamente um catalisador para a produção de artigos de qualidade no país. 


\section{RDBCI}

\section{RESULTADOS E DISCUSSÃO}

Os dados obtidos a partir das fontes já mencionadas, e verificados tanto na Web of Science como nas bases de dados SCOPUS, deram os seguintes resultados, que serão apresentados como porcentagem no quadro 1. Poderemos também apreciar a variação e diferença entre os países que compõem a América Latina e o impacto das suas revistas indexadas tanto na WoS como na SCOPUS (Gráfico 1).

Quadro 1. Revistas latino-americanas indexadas nas bases de dados WoS e Scopus.

\begin{tabular}{cccll}
\hline PAISES LATINO- & \multicolumn{2}{c}{$\begin{array}{c}\text { BASE DE DADOS DE } \\
\text { ALTO IMPACTO }\end{array}$} & VALOR PERCENTUAL \\
AMERICANOS & WOS & SCOPUS & WOS & SCOPUS \\
\hline ARGENTINA & 150 & 64 & 11,59 & 7,68 \\
BRASIL & 533 & 378 & 41,19 & 45,38 \\
CHILE & 108 & 102 & 8,35 & 12,24 \\
COLÔMBIA & 212 & 99 & 16,38 & 11,88 \\
CUBA & 29 & 25 & 2,24 & 3,00 \\
EQUADOR & 20 & 2 & 1,55 & 0,24 \\
MÉXICO & 126 & 114 & 9,74 & 13,69 \\
PERU & 29 & 9 & 2,24 & 1,08 \\
URUGUAI & 24 & 1 & 1,85 & 0,12 \\
VENEZUELA & 63 & 39 & 4,87 & 4,68 \\
TOTAL / AMÉRICA LATINA & 1294 & 833 & 100,00 & 100,00 \\
\hline Fonte: Elaboração própria do investigador. & & &
\end{tabular}

Graph 1. Resultados do número total de Revistas latino-americanas indexadas na base de dados WOS e SCOPUS.

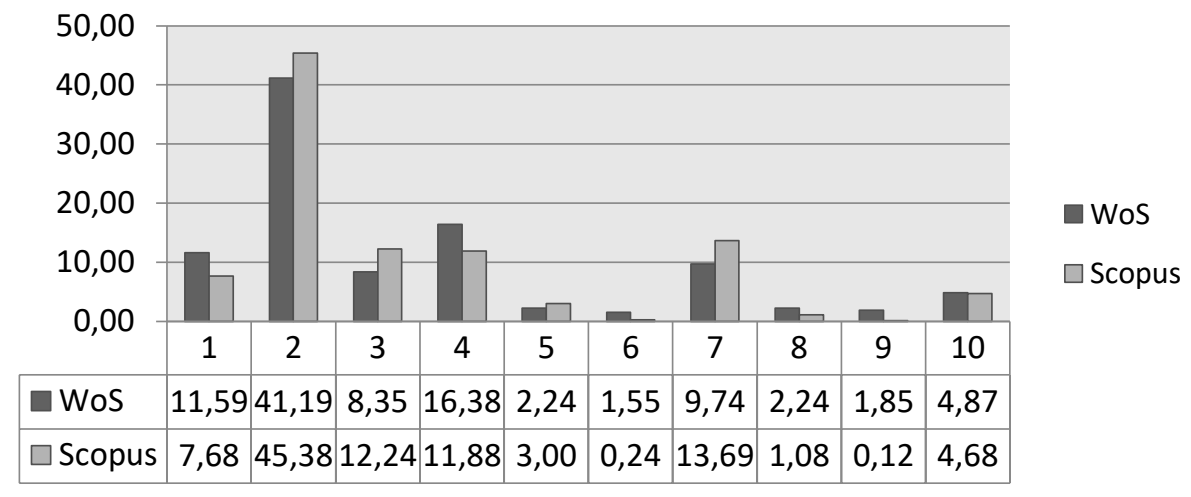

Fonte: Elaboração própria do investigador.

\subsection{Impacto das publicações latino-americanas em revistas indexadas internacionalmente}

No período da pesquisa de 2012 a 2018, foi observado um aumento significativo na quantidade de revistas latino-americanas indexadas em SJR e JCR, embora este número ainda represente uma porcentagem muito baixa da produção científica mundial. Da mesma forma, na América Latina, o número de revistas indexadas ao JCR equivale a 3,5\% da produção mundial. 


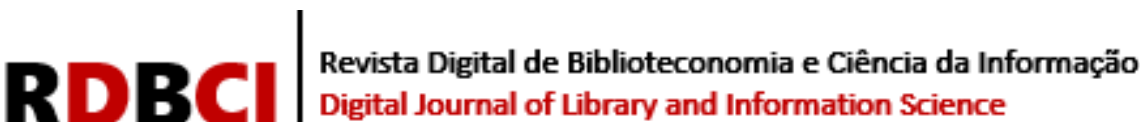

Assim, para o SJR e dentro da categoria de Ciências do Ambiente, o número médio de revistas latino-americanas indexadas equivale a 2,36\% da produção mundial (CRESPO et al., 2019). Esta investigação confirma os resultados do estudo de SANCHO et al. (2006), que mostrou que, embora a América Latina tenha a maior biodiversidade do mundo e um vasto território, a sua proliferação científica no campo da pesquisa, nas principais bases de dados (WoS e Scopus) ainda conserva um baixo nível percentual em comparação com o resto do mundo, apresentando assim as suas revistas indexadas, um nível de impacto inferior à média das outras regiões. Do mesmo modo, estes resultados reforçam o que RODRÍGUEZ-MORALES (2018) afirma que a América Latina deve continuar a fazer um esforço para gerar revistas científicas de elevado rigor científico e excelente qualidade, que poderiam ser indexadas na base de dados de alto impacto, gerando um vasto stock de revistas, onde os autores da região poderiam publicar as suas pesquisas, já que a maioria destes estudos mostrou claramente o principal obstáculo que estes investigadores têm para conseguir a publicação dos seus artigos em revistas de grande impacto e isto é uma consequência do facto de muitos editores ignorarem os principais problemas locais ou a língua da investigação (MAMMIDES et al. , 2016).

\subsection{Impacto das publicações latino-americanas em revistas indexadas internacionalmente}

As cifras internacionais têm refletido um notável desenvolvimento econômico para alguns países da América Latina nos últimos anos. O Peru, com base nos resultados dos seus indicadores macroeconômicos, tem sido um dos países que recentemente tem sido utilizado como modelo para tais progressos, ultrapassando a taxa média de 5\%, desde 2015 até a atualidade. Este auge expansivo foi objetivamente comprovado, através da sua consistência na análise da produção científica das suas próprias revistas. Se deve destacar que, no Peru, uma das suas principais desvantagens é que a maioria dos seus investigadores profissionais e publicadores de alto impacto estão fora do país. Além disso, a diferença entre as universidades privadas e públicas faz crescer esta diferença de produtividade no país, em relação ao resto dos países latino-americanos.

O Peru, de acordo com os dados do SCOPUS, registrou como um dos países com maior colaboração internacional, além disso as suas publicações são de grande impacto, pelo que a questão permanece: Porque o Peru não consegue posicionar-se melhor neste ranking? Ou porque o Peru não indexa mais revistas nestas bases de dados? Isto obviamente gerou uma baixa produtividade. Atualmente, o país está passando por uma reforma universitária, portanto, no âmbito da transformação universitária e dos processos que isso implica, o Ministério da Educação assinou um acordo com a CONCYTEC, para aumentar a competitividade do país através do reforço da capacidade investigativa e do fortalecimento dos programas de pesquisa. Entretanto, a universidade peruana ainda requer uma análise da infraestrutura e capacidade de seus recursos humanos (Medina Coronado, 2018), a fim de enfatizar a geração de novos conhecimentos, através da pesquisa e da publicação de artigos em revistas peruanas como prioridade e, assim, gerar uma melhor posição para o país no ranking das bases de dados WOS e SCOPUS.

\subsection{Impacto das publicações equatorianas no contexto global das revistas indexadas internacionalmente}

Certamente, o Equador não tem sido prolífico em termos de pesquisa científica, pelo que não foi surpreendente que durante o período 2012-2018, a sua produção científica tenha 


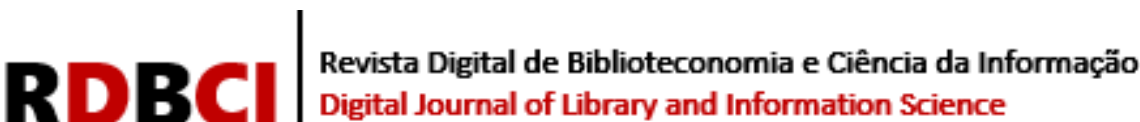

sido tão mínima. De acordo com a base de dados SCOPUS, o total de publicações do país representou um número igual a 6.548 e grande parte destas foram realizadas por investigadores ligados a organizações equatorianas. Um total de $89 \%$ dos documentos foram publicados em inglês e os $11 \%$ restantes, em espanhol ou outros idiomas, o que representou um aumento de 5,1 vezes e isto em comparação com o crescimento médio latino-americano das publicações, destacando-se dos restantes países, de acordo com relatórios até 2018. A taxa de crescimento anual composta (CAGR), também registrou que o Equador, posicionou-se atualmente como o país mais desenvolvido em termos de publicações científicas $(17,7 \%)$, seguindo as etapas que se localizam, Colômbia (14,8\%), Peru (11,2\%), Brasil (6,6\%), Argentina (5,2\%) e México $(4,6 \%)$. Os pesquisadores desenvolveram maiores avanços nas duas áreas especificamente, Agricultura - Ciências Biológicas e Medicina, que abrangeram dentro do conjunto, um total de $29 \%$ e $28 \%$ das publicações respectivamente (CASTILLO e POWEL, 2019). Uma das principais causas deste crescimento atual no sector da investigação que o país tem apresentado, especialmente na última década, deve-se fundamentalmente às mudanças, que foram levadas a cabo, na política governamental estabelecida. A Constituição do Equador (atualizada em 2008) estabeleceu que tanto o país como o governo eram obrigados a dar prioridade à educação com elevados padrões de qualidade, ligando fortemente este aspecto ao papel desempenhado pela pesquisa científica (Artigos 385-388, Constituição Política do Equador). Em 2010, a Lei Orgânica do Ensino Superior (LOES) foi decretada e estabelecida, e com isso, conseguiu-se uma promoção adequada no campo da investigação nos centros universitários, insistindo principalmente que todos os professores desses estabelecimentos devem ter pelo menos um mestrado e outra cláusula indicando que 70\% deles estavam sujeitos à apresentação de um nível de doutorado, que deve ser acreditado pelos centros de investigação universitários (MEDINA et al., 2016).

Neste contexto, o aumento da produção científica no Equador foi promovido através de dois programas, que contribuíram significativamente e que são: o Programa de Bolsas de Estudo e o Projeto Prometeo. O governo nacional, que ainda procurava melhorias no sector da produção científica nacional, gerou uma nova lei, chamada " Código Ingenios ", que foi aprovada e decretada em dezembro de 2016. Esta disposição promove, regula e financia o desenvolvimento tecnológico, a criatividade e a investigação científica, bem como obter a regulamentação na reprodução, gestão correta e exploração econômica da propriedade intelectual (patentes, protótipos, etc.). Deve também ser mencionado que a colaboração científica internacional na produção de publicações equatorianas tem gerado um impacto significativo, representando $80 \%$ da produção científica total e, embora a cooperação internacional tenha sido uma medida promovida pelos países desenvolvidos, este modelo muitas vezes não dá prioridade às necessidades dos países em desenvolvimento (PATALANO, 2005), embora existam atualmente grandes avanços no sector da produção científica no Equador, ainda este país enfrenta grandes desafios a resolver, principalmente em termos da qualidade das suas publicações indexadas.

\section{CONCLUSÕES}

A presente pesquisa foi concluída levando em conta os resultados obtidos na revisão sistemática das referências, sobre publicações de alto impacto, colocadas em revistas indexadas a bases de dados de alto nível, tais como: SCOPUS e Web of Science (WOS), que tanto o Peru quanto o Equador, ainda estão a se desenvolverem no campo da produção científica, em comparação com o contexto global, uma vez que a base de dados que atualmente tem maior impacto, é SCOPUS, onde o Peru tem apenas 4 revistas indexadas e o Equador somente 2 


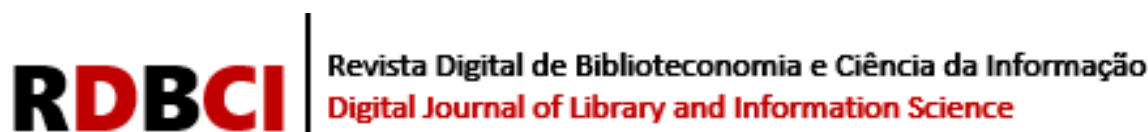

revistas. Isto significa que as políticas públicas conseguiram algumas melhorias no sector da investigação de cada país, respectivamente, mas estes esforços ainda não são suficientes para posicionar ambos os países numa boa posição no ranking da base de dados SCIMAGO/SCOPUS. Por conseguinte, é necessário que tanto o governo como as entidades privadas continuem a apoiar este sector de investigação e publicação em ambos os países, uma vez que, de acordo com os dados consultados, a economia nacional está fortemente ligada ao progresso científico do país envolvido.

Finalmente, é evidente que o caminho de cada país é aumentar a produtividade, mas no caso específico do Equador, que esteve tão longe da investigação durante tantos anos, é importante compensar esta sequela ocasionada e não só aumentar o seu impacto nas bases de dados WOS e SCOPUS, mas também posicionar mais revistas em SCOPUS, é muito importante que a qualidade das suas publicações seja melhorada.

CRediT
REONHECIMENTOS: Não aplicável.
FINANCIAMENTO: Não aplicável.
CONFLITOS DE INTERESSES: Os autores certificam que não têm interesse comercial ou associativo que represente
um conflito de interesses em relação ao manuscrito.
APROVAÇÃO DE ÉTICA: Não aplicável.
DISPONIBILIDADE DE DADOS E MATERIAIS: Não aplicável.
CONTRIBUIÇÕESDOS AUTORES: Conceituação, Curadoria de Dados, Análise Formal, Aquisição de Financiamento,
Investigação, Metodologia, Administração de Projetos, Recursos, Software, Supervisão, Validação, Visualização,
Redação - rascunho original: Israel Barrutia B.; Conceituação, Curadoria de Dados, Análise Formal, Aquisição de
Financiamento, Investigação, Metodologia, Administração de Projetos, Recursos: Haiber Policarpio Echevarria
Rodríguez; Recursos, Software, Supervisão, Validação, Visualização, Redação: Wilmer Ortega Chávez.

\section{REFERÊNCIAS}

ANGULO, R. et al. Student Publication in Venezuelan Medical Journals, 2001 -

2005. Ciencia e Investigación Médica Estudiantil Latinoamericana, v. 13, n.1, p. 6-8. 2008. Disponível em: http://www.cimel.felsocem.net/index.php/CIMEL/article/view/156. Acesso em: 22/01/2019.

CASTILLO, J.; POWELL, M. Analysis of the scientific production of Ecuador and the impact of international collaboration in the period 2006-2015. Revista española de Documentación Científica, v. 42, n.1, p. 12-25. 2019. doi: 10.3989/redc.2019.1.1567

CASTRO-RODRÍGUEZ, Y. Student scientific production in Peruvian dental journals during the period 2012 to 2017. Educación Médica, v. 20, n. 2, p. 91-99. 2019. doi: $\underline{10.1016 / j . e d u m e d .2018 .02 .008}$

COCCIA, M.; ROLFO, S. How changes in research policies can affect the organization and productivity of public research institutes: an analysis within the Italian national innovation system. Journal of Comparative Policy Analysis: Research and Practice, v. 9, n. 3, p. 215-233. 2007. doi: $\underline{10.1080 / 13876980701494624}$ 


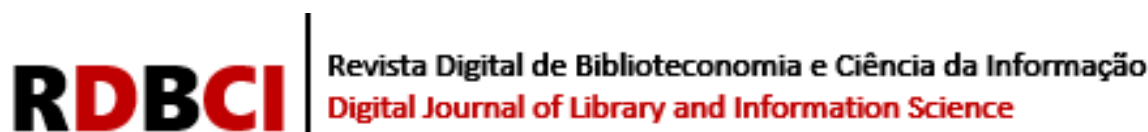

CRESPO-GASCÓN, S. et al. Production of scientific journals in Latin America and the Caribbean in Scopus, Journal Citation Reports and Latindex in the area of natural resources: their relationship with economic, environmental, and research investment variables. Revista Española de Documentación Científica, v. 42, n. 1, p. 12-24. 2019. doi: $10.3989 /$ redc.2019.1.1533

CRUZ-RAMÍREZ, M. et al. Scientometric analysis of Cuban educational publications in WoS and Scopus (2003-2012). Revista Española de Documentación Científica, v. 37, n. 3, p. 43-58. 2014. doi: 10.3989/redc.2014.3.1119

DRUCKER, J.; GOLDSTEIN, H. Assessing the impacts of regional economic development of universities: a review of current approaches. International Regional Science Review, v. 30, n. 1, p. 20-46. 2007. doi: $10.1177 / 0160017606296731$

ESTRADA-CUZCANO, A.; ALFARO, K. Scientific production of researchers in Humanities and Social Sciences of the Universidad Nacional Mayor de San Marcos: 2017-2018. 2019. Disponível em: https://n9.cl/69gp. [18 de enero de 2019]. http://revista.letras.unmsm.edu.pe/index.php/itc/article/view/639. Acesso em: 22/01/2019.

GARCIA-BEREGUIAIN, M. Journals indexed on Scopus in Ecuador: the pending subject. Revista Ecuatoriana de Neurología, v. 28, n. 3, p. 13-14. 2019. Disponível em: http://scielo.senescyt.gob.ec/scielo.php?script=sci_arttext\&pid=S2631-25812019000300013. Acesso em: 22/01/2019.

GONZÁLEZ-ARGOTE, J. et al. Student scientific production in Cuban medical journals 1995-2014 First stage in Research. Educación Médica, v. 5, n. 19, p. 155-163. 2016. doi: $10.1016 /$ j.riem.2016.01.023

GUERRA, K. et al. Bibliometric analysis of the publications related to innovation projects and their management in Scopus, in the period 2001-2011. ACIMED, v. 24, n.3, p. 281-294. 2013. Disponível em: https://www.medigraphic.com/cgibin/new/resumen.cgi?IDARTICULO=45749. Acesso em: 22/01/2019.

HUAMANÍ, C. et al. Student contribution in the publication of scientific articles in medical journals indexed in SciELO-Peru, 1997 - 2005. Anales de La Facultad de Medicina, v. 69, n. 1, p. 42-45. 2013. Disponível em: http://www.scielo.org.pe/scielo.php?pid=S102555832008000100009\&script=sci_arttext\&tlng=en. Acesso em: 22/01/2019.

MARDONES, R. Discourse of the territory enunciated by the Social Sciences in the scientific publications of Latin American journals. Revista Prisma Social, n. 16, p. 556-596, 23 dic. 2016. Disponível em: https://revistaprismasocial.es/article/view/1266. Acesso em: 22/01/2019.

MAMMIDES, C. et al. Increasing geographic diversity in the international conservation literature: A stalled process? Biological Conservation, n. 198, p. 78-83. 2016. doi: $10.1016 /$ j.biocon.2016.03.030

MEDINA, J. et al. Scientific research, in Ramírez René [comps.]. Universidad urgente para una sociedad emancipada, p 461-494. Quito, Ecuador: SENESCYT-IESALC. 2016. 


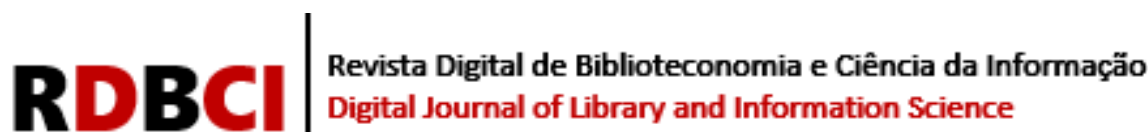

MOREIRA-MIELES, L. et al. Characterization of Ecuador's scientific production in the period 2007-2017 in Scopus. Investigación Bibliotecológica: Archivonomía, Bibliotecología e Información, v. 34, n. 82, p. 141-157. 2020.

doi: 10.22201/iibi.24488321xe.2020.82.58082.

PATALANO, M. Publications in the scientific field: the academic journals of Latin America. Anales de Documentación: Revista de Biblioteconomía y Documentación, v. 8, n. 8, p. 217-235. 2005. Disponível em: https://revistas.um.es/analesdoc/article/view/1451. Acesso em: 22/01/2019.

PERDOMO, B. et al. Quality of undergraduate theses in Peruvian universities. Revista Espacios, v. 41 n. 2, p. 5-17. 2020. Recuperado de Disponível em: https://www.revistaespacios.com/a20v41n02/20410205.html. Acesso em: 22/01/2019.

RAIHER, D. Comparative study between Latin American and world scientific production on health inequities during the years 1999-2008. (Bachelor Thesis) National University of La Plata. 2010. Available at: sedici.unlp.edu.ar. Disponível em: http://www.memoria.fahce.unlp.edu.ar/library?a=d\&c=tesis\&d=Jte361. Acesso em: 22/01/2019.

RIOS-GONZÁLEZ, C. Scarce scientific publication in Paraguayan medical students. Educación Médica, v. 17, n. 2, p. 80-81. 2016. doi: 10.1016/j.edumed.2016.03.005.

RODRÍGUEZ-MORALES, Publish from Latin America. Where are we? Revista Ecuatoriana de Neurología, v. 27, n. 3. 2018. Disponível em: http://scielo.senescyt.gob.ec/ scielo.php?script=sci_arttext\&pid=S2631-25812018000300012. Acesso em: 22/01/2019.

SANCHO, R. et al. Indicators of scientific inter-center collaboration in Latin American countries. Interciencia, v. 31, n. 4, p. 284-292. 2006. Disponível

em: https://www.redalyc.org/pdf/339/33911508.pdf. Acesso em: 22/01/2019.

SCIMAGO. SCimago Lab: Scientific excellence georeferenced. The neighborhood matters. 2011. Disponível em: http://www.scimagolab.com/blog/2011/scientific-excellencegeoreferenced-the-neighborhood-matters. Acesso em: 22/01/2019.

TAYPE-RONDÁN, Á. et al. Student scientific production in Latin America: an analysis of Spanish-language medical journals indexed in SciELO, 2011. Educación Médica, v. 17, n. 3 , p. 171-177. 2014. doi: $10.4321 /$ S2014-98322014000300007

TEJEDOR-ESTUPINAN, J. Y TEJEDOR-ESTUPINAN, R. Visibility and scientific impact: the case of Colombian economy journals. Investigación Bibliotecológica: Archivonomía, Bibliotecología e Información, v. 33, n. 78, 121-141. 2019. doi: $10.22201 /$ iibi.24488321xe.2019.78.57915.

VALLE, R. y SALVADOR, E. Bibliometric analysis of the undergraduate thesis of the Faculty of Medicine of the Nacional University of San Marcos. Anales de La Facultad de Medicina, v. 70, n. 1, p. 11-18. 2012. Disponível em: http://www.scielo.org.pe/scielo.php?pid=S1025-55832009000100003\&script=sci _arttext\&tlng=en. Acesso em: 22/01/2019. 


\section{RDBCI|}

VERA-MONGE, V. et al. Latin American Scientific Production in Health Economics, 20052014. Salud Pública De México, n. 59, p. 505-506. 2017. Disponível em: https://www.scielosp.org/article/spm/2017.v59n5/505-506/. Acesso em: 22/01/2019.

ZACCA-GONZÁLEZ, G. et al. Latin American scientific production in public health: combined analysis using bibliometric, socioeconomic and health indicators. Scientometrics, v. 102, n. 1, p. 609-628. 2015. Disponível em: https://dialnet.unirioja.es/servlet/ tesis?codigo $=57310$. Acesso em: 22/01/2019.

ZUÑIGA, U. University and Science in Latin America. Interface - Comunicação, Saúde, Educação, v. 4, n. 6, p. 167-170. 2000. Disponível em: https://www.scielosp.org/article/icse/ 2000.v4n6/167-170/es/. Acesso em: 22/01/2019. 


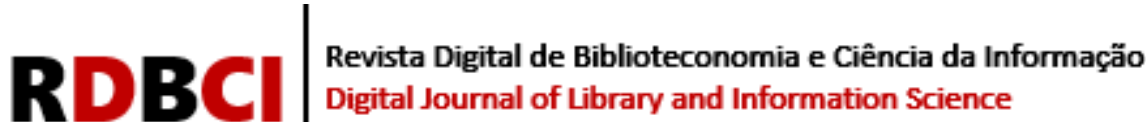

